\title{
ACOMPAÑAMIENTO DOCENTE COMO MEDIADOR ENTRE LA COMUNIDAD DE ESTUDIO Y LA ESCRITURA ACADÉMICA
}

\author{
Consuelo del Rocío Pérez ${ }^{1^{*}}$ \\ ${ }^{1}$ Pontificia Universidad Católica del Ecuador, Facultad de Ciencias de la Educación \\ * Autor para correspondencia: cperez502@puce.edu.ec \\ Recibido: 2020/03/05 Aprobado: 2020/11/25 \\ DOI: https://doi.org/10.26621/XVI23.2020.12.A02.PUCESI.2550.6684
}

\section{RESUMEN}

Frente a la problemática presente en el desempeño escrito de los estudiantes universitarios ecuatorianos, este estudio pretende estimar el grado de desarrollo de competencias escriturales propias de los géneros discursivos académicos. El diseño del estudio es descriptivo. La muestra estuvo compuesta de 58 escritos de estudiantes de una universidad pública ecuatoriana. Estos escritos son analizados a través de la estructuración de una rúbrica con criterios de valoración que se consideran fundamentales en la redacción académica. El estudio revela un nivel escritural bajo, lo que permite concluir que los estudiantes no han sido alfabetizados académicamente, situación que involucra la figura docente.

Palabras clave: alfabetización académica, acompañamiento docente, competencias escriturales, género discursivo

\section{ABSTRACT}

In view of the problems present in the written performance of Ecuadorian university students, this study aims to estimate the degree of development of writing skills typical of academic discursive genres. The design of the study is descriptive. The sample was composed of 58 writings by students from an Ecuadorian public university. These writings were analyzed through the structuring of a rubric with evaluation criteria that they are considered fundamental in academic writing. The study reveals a low level of writing, which allows us to conclude that the students have not been academically literate, a situation that involves the teaching figure.

Keywords: academic alphabetization, teacher accompaniment, writing skills, discursive gender 


\section{INTRODUCCIÓN}

Investigaciones sobre el nivel de las competencias escriturales de los estudiantes universitarios cobran mucha importancia en la actualidad. Estudios de Guzmán y García (2015), Fregoso (2011), Carlino (2013, 2008, 2018), Sorókina (2008), Andueza y Aguilera (2018), González (2003), entre otros, dan cuenta de esta problemática. En este sentido, la teoría de la alfabetización académica establece que el estudiante necesita ser inducido en la universidad en la redacción de los géneros discursivo-científicos que requiera para producir sus escritos. En América Latina, esta teoría ha tenido eco principalmente por la figura de Carla Paulino, quien deja en claro que la academia tiene su propia alfabetización en redacción, criterio compartido con Núñez (2013) quien establece que la educación es un continuum, y al ser la redacción producto de procesos cognitivos, también implica un estudio y desarrollo constante en el ser humano.

\section{Alfabetización académica y acompañamiento docente}

La alfabetización académica no se restringe tan solo a aprender a leer y escribir; más bien, hace alusión a la alfabetización escrita como cultura de transmisión de conocimiento (Barriga y Viveros, 2009, citados en Núñez, 2013), por lo que propende al desarrollo de habilidades cognitivas de cada disciplina a través de la lectura y la redacción (Carlino, 2013). En este marco, la alfabetización académica desarrolla las competencias necesarias para participar en la cultura discursiva y en una comunidad de aprendizaje situada, corresponde a la una y a la otra "el ámbito académico superior" (Carlino, 2008).

Por ende, las capacidades escriturales de un estudiante universitario aún tienen mucho por madurar; por tanto, al docente le corresponde "enseñar procesos y prácticas discursivas de pensamiento" (Carlino, 2008), pues de no hacerlo niega la participación de su estudiante en la cultura discursiva académica respectiva a través de la producción de textos complejos que deben ser guiados por quienes dominan su respectiva disciplina (el docente), a fin de asegurar la comprensión de los conceptos que atañen a la misma. Por tanto, no se trata simplemente de técnicas de redacción que entrenen a los estudiantes y que les permitan cumplir su trayectoria de estudiantes -es decir, cumplir por cumplir-; se trata del desarrollo cognitivo y apropiación de conceptos, de una apropiación epistémica del lenguaje (Molina, 2012), como autorregulación para participar en una actividad social cognitiva y no un mero producto textual.

La redacción dentro de la teoría en análisis requiere de un alfabeto funcional, quien debe transferir. Esta operación cognitiva define la capacidad de reaplicación de la cognición en situaciones diferentes, lo cual permite, desde la base del conocimiento proponer soluciones a su contexto inmediato, divulgadas desde la escritura, que es lo que se espera de un estudiante universitario. Al no lograr transferir, cualquier aprendizaje es considerado como una repetición de algo conocido, pero mutilado por la incapacidad de transformación y creación (Perrenoud, 2007).

Guzmán y García (2015) estudiaron las experiencias de profesores y estudiantes sobre alfabetización, y las estrategias con las que la llevan a la práctica. Uno de sus resultados fue que tanto un grupo como el otro no logran unas buenas prácticas que incidan en una óptima redacción académica por ausencia de un proceso situado de lectura y escritura críticas. Esto radica en que la propia comunidad universitaria no estructura un modelo de alfabetización de acuerdo con su contexto y sus especificidades desde tres dimensiones: las competencias escriturales de las que deben ser capaces los estudiantes, las necesidades académi- cas de la institución y un modelo específico de alfabetización académica (Guzmán y García, 2015; Ávila, González y Peñaloza, 2013). Vista así, la redacción constituye un proceso que no se desarrolla naturalmente, sino que es el contexto universitario el encargado de alfabetizar directamente a sus estudiantes.

En consecuencia, se puede entender que el estudiante no puede transitar solo en la redacción académica, pues debe comprender el contexto social, cultural y geográfico en el que se encuentra (Loureda, 2010), además de la comunidad de discurso a la que pertenece (Vargas, 2016). En este marco, necesita del docente disciplinar ya que en cada práctica de redacción requiere apropiarse de la episteme de las diferentes cátedras (Molina, 2012), lo que convierte tanto a la redacción como a la episteme en práctica y lenguaje situados.

Fregoso (2011), a través de un estudio de caso realizado en estudiantes de licenciatura y maestría, reveló deficiencias a nivel ortográfico, morfológico, semántico y sintáctico. Al respecto, se puede indicar que en una comunidad académica los problemas en redacción van más allá de la gramática normativa porque los géneros discursivos son especializados y con base en el pensamiento abstracto y divergente (Rojas, 2016); así, la semántica atañe a la retórica del conocimiento científico o epistemológico (Hernández, 2009). Tal situación determina la redacción como una habilidad epistémica (Núñez, 2013) que necesita de la alfabetización gradual del docente en prácticas discursivas, porque el género a través de la redacción se constituye en un constructo cognitivo-evolutivo inherente al sujeto de tal manera que posibilita su actuación en diferentes contextos (Parodi, 2008).

\section{Las competencias escriturales y el género discursivo}

El género discursivo, al estar inmerso en una comunidad de aprendizaje, es un proceso social que concreta la divulgación del saber; por tanto, comunica resultados que tienen un propósito: llegar a un campo científico (Alexopoulou, 2011) organizado en etapas (Placci y Garófolo, 2014). Visto así, se puede decir que el discurso es una unidad comunicativa con un objetivo específico y un contexto de situación dado (Gil, 1999), entendido este último como la situación particular de un grupo social llamado universidad (Rojas, 2016). Sin embargo, desarrollar la capacidad discursiva de un estudiante exige competencias escriturales (pertinentes para generar discursos) de un docente experto en una disciplina específica y de un docente experto en lenguaje: así, son tres condiciones que ponen en franco riesgo la redacción académica debido a que tal trilogía debe llegar a un consenso para obtener un solo producto: divulgación científica escrita.

Dentro de las competencias escriturales que requiere conocer el estudiante, a más del conocimiento epistémico disciplinar, es mantener la progresión temática (Gil, 1999) a través de la tesis, pues en los textos científicos existe una construcción y reconstrucción de ideas unidas por el tema (Cantillo y Vargas, 2015). La progresión temática ha sido estudiada de forma más frecuente y es la que mayor grado de efectividad mantiene para lograr la coherencia global, elemento fundamental para desarrollar el objetivo comunicacional, es decir, la interconexión entre escritor y lector. Al respecto, en los estudios realizados por Villavicencio et al. (2018) en estudiantes de una universidad pública ecuatoriana, uno de los problemas detectados en la redacción académica fue mantener la coherencia global, lo que hace imposible, a decir de los autores, entender los escritos de los estudiantes. Tal situación se puede entender porque el texto no es una suma de enunciados, sino que es una unidad total -cohesión semántica-, o sea, es una composición de vínculos 0 dependencias intertextuales, no solo interoracionales (Sorókina, 2008). 
En el desarrollo de la coherencia global se encuentra implícita la coherencia lineal semántica, aquella que se sitúa en los párrafos y establece relaciones entre conceptos, proposiciones y subtemas, y de esta manera construye el aspecto temático-ideológico que determina la identidad cognitiva entre emisor y receptor ${ }^{1}$ para llegar a un razonamiento susceptible de explicar la intención global que conlleva el texto y hacerlo accesible (Andueza \& Aguilera, 2018). Este tipo de coherencia debe ser evidente en la introducción, desarrollo y conclusión del párrafo, debido a que se evita la digresión y se establece una relación de reciprocidad (Sorókina, 2008), en consecuencia es el párrafo la base de la coherencia global, y de la función expositivo-argumentativa que sostiene el texto y lo constituye en una unidad de sentido y de pensamiento (Álvarez, 2005; Loureda, 2010). En tal sentido, la redacción debe estar en comunión entre su "carácter comunicativo 0 de significación, social o de contexto de situación, pragmático 0 propósito del emisor, su coherencia semántica y su carácter semántico y global" (Iglesias, González y Hernández, 2019, 171 y 172).

No obstante, Padilla et al. (2011) consideran que el problema en competencias escriturales tiene base en la argumentación. Afirman que los estudiantes no respaldan sus opiniones con fuentes, lo que remite a un problema más grave: falta de lectura e investigación (Moore y Velásquez, 2013). Tal situación deriva en la ausencia de intertextualidad, competencia escritural medular para no redactar con juicios de valor, elemento que no tiene cabida en una comunidad discursiva académica. A través de la argumentación se conquista una determinada comunidad científica 0 un grupo con características definidas, las que deben ir en consonancia con la producción escrita (Díaz, 2009). Un texto que no mantiene y no desarrolla la intertextualidad ("dialogicidad" para Bajtín, 2011; "polifonía textual" para Adoumieh, 2018) es un texto acrítico 0 una simple compilación de otros textos, pues la voz del escritor no se escucha, lo que implica falta de experiencia investigativa; por ende, el texto carecerá de originalidad. En este sentido, es necesario remitirse a las voces de los otros (Cohen, 2007), pero con la intención propia del escritor para que su voz tenga eco (Polo, 2012). En consecuencia, la intertextualidad y su respectivo respaldo a través de las diferentes normativas de citación es clara evidencia de estar informado sobre el tema, esto es, que el conocimiento propio empezó a construirse a través del de los demás (Karam, 2005).

Un estudiante alfabetizado no incurre en la simple compilación o en el peor de los casos, en el plagio. En el periódico local El Universo (14 de noviembre de 2013) se presentó el siguiente titular: Se presume plagio en la mitad de tesis de universitarios; René Ramírez, el entonces encargado de la Secretaría Nacional de Educación Ciencia, Tecnología e Innovación (Senescyt), tiempo atrás afirmó que, entre 2010 y 2013, "al menos una de cada dos tesis son plagios o puede constituir en un fraude académico"; esta situación es crítica al tener en cuenta que la tesis es un género discursivo específico de la academia (Borsinger De Montemayor \& al., 2014), género que evidencia un desarrollo cognitivo-científico no solo de los estudiantes, también de quienes dirigen la educación de las comunidades de aprendizaje.

En definitiva, la alfabetización académica propende a desarrollar en el estudiante, a más de las competencias escriturales, la autorregulación de sus aprendizajes a través de la metacognición, del pensamiento lógico y crítico (Mestanza, 2018; Adoumieh, 2018). En consecuencia, la presencia del docente de las respectivas disciplinas es fundamental, pues él es el único que puede inducir a sus estudiantes a la apropiación epistémica disciplinar y revertirla en una producción escrita coherente, argumentada, intertextualizada y crítica desde el currículo (Carlino, 2008).

\section{MATERIALES Y MÉTODOS}

Este estudio pretendió determinar, desde el análisis de la redacción académica de los estudiantes, si es necesario el acompañamiento del docente de cada disciplina en el desarrollo de sus producciones escritas. A través de un diseño descriptivo, se analizaron las variables dependiente e independiente y se obtuvieron los datos estadísticos correspondientes a la media, mediana y moda de las competencias escriturales de la muestra. El análisis se sujetó a dos criterios de valoración: la coherencia local y la coherencia global que deben presentar los textos académicos. Su selección se debió a que son una parte fundamental que marca la madurez en la escritura, al igual que el grado de apropiación epistémica de las diferentes disciplinas por parte de los estudiantes. En la Tabla 1 se esquematizan las variables del estudio.

Tabla 1: Variables en estudio

\begin{tabular}{|c|c|c|}
\hline Variable dependiente & Escala de valores & $\begin{array}{c}\text { Criterios } \\
\text { involucrados }\end{array}$ \\
\hline \multirow{3}{*}{$\begin{array}{l}\text { Coherencia local } \\
\text { (párrafos) "argumen- } \\
\text { tos" }\end{array}$} & $\begin{array}{l}0=(\text { All })=\text { argumenta- } \\
\text { ción incoherente } 0 \text { inexis- } \\
\text { tente }\end{array}$ & \multirow{3}{*}{$\begin{array}{l}\text { Intertextualidad } \\
\text { Normativa de } \\
\text { citación } \\
\text { Escritura episté- } \\
\text { mica }\end{array}$} \\
\hline & $\begin{array}{l}1=(\mathrm{APCC})=\text { argumentos } \\
\text { parcialmente críticos y } \\
\text { coherentes }\end{array}$ & \\
\hline & $\begin{array}{l}2=(\mathrm{ACC})=\text { argumentos } \\
\text { críticos y coherentes }\end{array}$ & \\
\hline Variable independiente & Escala de valores & $\begin{array}{c}\text { Criterios } \\
\text { involucrados }\end{array}$ \\
\hline \multirow{3}{*}{$\begin{array}{l}\text { Coherencia global } \\
\text { (textual) "Tesis" }\end{array}$} & $\begin{array}{l}0=(T I I)=\text { totalmente in- } \\
\text { coherente } 0 \text { inexistente }\end{array}$ & \multirow{3}{*}{$\begin{array}{l}\text { Progresión temá- } \\
\text { tica } \\
\text { Género discursivo } \\
\text { Metacognición }\end{array}$} \\
\hline & $\begin{array}{l}1=(\mathrm{TPC})=\text { tesis parcial- } \\
\text { mente coherente }\end{array}$ & \\
\hline & $2=(\mathrm{TC})=$ tesis coherente & \\
\hline
\end{tabular}

A través de una fórmula estadística, se obtuvo el tamaño de la muestra del periodo correspondiente 2019:

$$
n=\frac{1,96^{2} * 0,5 * 0,5 * 170}{0,105^{2}(170-1)+1,96^{2} * 0,5 * 0,5}=57,82 \approx 58
$$

Durante la redacción de los textos analizados, no hubo intervención del docente en ningún momento; ello permitió establecer el nivel de redacción natural, es decir, sin intervención o mediación de ninguna clase. También se especificó que elijan un tema de su carrera; esta indicación fue medular, pues al estar entre cuatro y cinco semestres en la carrera respectiva, la muestra fue idónea para determinar la apropiación epistémica disciplinar. El instrumento con el que se analizaron los ensayos fue una rúbrica estructurada con base en el referente teórico expuesto (en la Tabla 1 se encuentran los respectivos criterios de valoración que tomaron el nombre de variables y criterios involucrados).

1 Ecured.cu. (9 de 10 de 2019). Obtenido de https://www.ecured.cu/Coherencia_sem\%C3\%A1ntica 


\section{RESULTADOS Y DISCUSIÓN}

A continuación, se presentan los datos y su respectivo análisis.

Tabla 2. Media, mediana y moda de la variable dependiente Coherencia Local (párrafos) "Argumentos"

\begin{tabular}{|c|c|c|}
\hline \multicolumn{3}{|c|}{ Estadísticos } \\
\hline \multicolumn{3}{|c|}{ Coherencia local (párrafos) Argumentos } \\
\hline \multirow[t]{2}{*}{$\mathrm{N}$} & Válido & 58 \\
\hline & Perdidos & 0 \\
\hline \multicolumn{2}{|c|}{ Media } & 0,71 \\
\hline \multicolumn{2}{|c|}{ Mediana } & 1,00 \\
\hline \multicolumn{2}{|c|}{ Moda } & 0 \\
\hline \multicolumn{2}{|c|}{ Desviación estándar } & 0,726 \\
\hline \multicolumn{2}{|c|}{ Varianza } & 0,527 \\
\hline
\end{tabular}

En la Tabla 2, se observa que los ensayos evaluados con la variable de la rúbrica denominada "Argumentos", tienen un valor de media (promedio) de 0,71 lo que significa que los ensayos evaluados tienen All 0 APCC. Mientras que el valor de mediana es de 1 que corresponde a una respuesta de APCC, y un valor de moda de 0 que corresponde a una respuesta de All.

Tabla 3. Porcentajes de la variable dependiente Coherencia Local "Argumentos"

Coherencia local (párrafos) Argumentos

\begin{tabular}{lcccc}
\hline & Frecuencia & Porcentaje & $\begin{array}{c}\text { Porcentaje } \\
\text { válido }\end{array}$ & $\begin{array}{c}\text { Porcentaje } \\
\text { acumulado }\end{array}$ \\
\hline $\begin{array}{l}\text { argumentación } \\
\text { incoherente 0 } \\
\text { inexistente (All) } \\
\text { argumentos par- } \\
\text { cialmente críticos }\end{array}$ & 26 & 44,8 & 44,8 & 44,8 \\
Válido & 23 coherentes \\
$\begin{array}{l}\text { (APCC) } \\
\text { argumentos críti- } \\
\text { cos y coherentes } \\
\text { (ACC) }\end{array}$ & 9 & 39,7 & 39,7 & 84,5 \\
Total & 58 & 15,5 & 15,5 & 100,0 \\
\hline
\end{tabular}

En la Tabla 3, se observa que los ensayos evaluados con la variable de la rúbrica denominada "argumentos" tienen un $44,8 \%$ de All y un $39,7 \%$ de APCC, lo que indica que los ensayos presentan argumentación incoherente 0 inexistente, con apenas un 15,5 de argumentos críticos y coherentes.

Estos porcentajes revelan que no se han desarrollado competencias escritoras necesarias para hacer frente a los géneros discursivos llevados en la universidad. De acuerdo con Guzmán y García (2015), es evidente la necesidad de la formación de los estudiantes en alfabetización académica, alfabetización respectiva a cada docente y a cada uno de los ámbitos del conocimiento. La situación se agrava al tener en cuenta que dentro de la argumentación es fundamental la intertextualidad para dotar de originalidad al texto y transmitir el punto de vista del escritor (Polo, 2012; Cohen, 2007). Si bien Karam (2005) y Moore y Velásquez (2013) consideran que la falta de intertexto es falta de investigación y lectura; también es evidencia de una franca falencia de argumentación coherente y crítica, que bien podría superarse a través de la lectura de textos relativos a los géneros discursivos manejados en la academia y contribuir a desarrollar estas competencias escriturales.
Tabla 4. Media, mediana y moda de la variable Independiente Coherencia global (textual) "Tesis"

\begin{tabular}{l|l|r}
\hline \multicolumn{2}{c}{ Estadísticos } \\
\hline $\mathrm{N}$ & Válido & 58 \\
\cline { 2 - 3 } & Perdidos & 0 \\
\hline Media & $\mathbf{0 , 6 6}$ \\
Mediana & $\mathbf{0 , 0 0}$ \\
Moda & $\mathbf{0}$ \\
Desviación estándar & 0,762 \\
Varianza & 0,581 \\
\hline
\end{tabular}

En la Tabla 4 se observa que los ensayos evaluados con la variable de la rúbrica denominada "Tesis", tienen un valor de media (promedio) de 0,66 lo que significa que los ensayos presentados muestran tesis TII o TPC; mientras que el valor de mediana es 0 que corresponde a una respuesta de tesis TII, y un valor de moda de 0 que también corresponde a una respuesta de tesis TII.

Tabla 5. Porcentajes de la variable independiente Coherencia Global (textual) "Tesis"

\begin{tabular}{lcccc}
\hline & Frecuencia & Porcentaje & $\begin{array}{c}\text { Porcentaje } \\
\text { válido }\end{array}$ & $\begin{array}{c}\text { Porcentaje } \\
\text { acumulado }\end{array}$ \\
\hline $\begin{array}{l}\text { totalmente } \\
\text { incoherente } \\
\text { o inexistente } \\
\text { (TII) }\end{array}$ & 30 & 51,7 & 51,7 & 51,7 \\
tesis par- & & & & \\
Válido & & & & \\
$\begin{array}{l}\text { cialmente } \\
\text { coherente } \\
\text { (TPC) }\end{array}$ & 18 & 31,0 & 31,0 & 82,8 \\
$\begin{array}{l}\text { tesis cohe- } \\
\text { rente (TC) }\end{array}$ & 10 & 17,2 & 17,2 & 100,0 \\
Total & 58 & 100,0 & 100,0 & \\
\hline
\end{tabular}

En la Tabla 5, se observa que los ensayos evaluados con la variable de la rúbrica denominada "Tesis" tienen un $51.7 \%$ de TII y un 31,0\% de TPC lo que indica que los ensayos presentan tesis totalmente incoherente 0 inexistente y un porcentaje de $17,2 \%$ de TC.

Los resultados expuestos son críticos y sin duda derivados de la falta de sistematización de ideas, proceso que tiene como función otorgar al escritor una idea general para desarrollar la progresión temática, a fin de construir el significado comunicacional del texto sin digresiones, de modo que el escrito esté dotado de unidad a través de su coherencia semántica y global (Iglesias, González y Hernández, 2019). Además, es necesario considerar que la falta de coherencia se relaciona directamente con la metarreflexión de los aprendizajes (Mestanza, 2018); en otras palabras, la autoconsciencia 0 autorregulación del propio aprendizaje. Además, es reflejo directo del desconocimiento de la estructura discursiva que la muestra debe manejar en la formación académica a través del desarrollo y argumentación de una tesis. En definitiva, la educación a lo largo de la vida o continuum (Núñez, 2013) no se concreta en este caso. 


\section{CONCLUSIONES}

De cara a los resultados expuestos a través de la variable dependiente, es evidente que los estudiantes necesitan de la dirección del docente para apropiarse de los procesos de redacción académica, ya que la argumentación incoherente 0 inexistente implica directamente a procesos de aprendizaje y de apropiación epistémica, que permiten al estudiante expresar su criterio y dejar sentada su voz a través de la argumentación.

Los resultados también revelan la falta de lectura y de conocimiento de autores que den la pauta al estudiante para argumentar y comentar fuentes o bases bibliográficas con sus docentes, situación que también deja en entredicho la figura docente y su responsabilidad frente al aprendizaje de sus estudiantes.

Los porcentajes en cuestión también traen a colación la imposibilidad de producir textos que divulguen el conocimiento científico al que debe propender cada disciplina; es decir, no existe una escritura crítica y epistémica en los estudiantes, pues la argumentación es evidencia de apropiación y generalización del conocimiento y parte fundamental del desarrollo de las competencias escriturales de los estudiantes.

Con relación al resultado obtenido de la variable independiente, el desarrollo de la progresión temática es débil; por ende, no existe coherencia global. También conduce a cuestionar si el estudiante está o no en un proceso de alfabetización académica, pues al mostrar evidencias de escaso desarrollo de esta competencia escritural tampoco evidencia indicios de metacognición que lo mantenga consciente de que párrafo a párrafo la progresión temática debe estar presente.

El resultado también deriva al desconocimiento de estructuras de géneros discursivos, ya que la progresión temática marca el género de la comunidad de estudio a la que pertenecen. Pero los géneros deben ser revelados a través de la alfabetización académica por medio de la figura del docente. Más aún, un estudiante que no sea alfabetizado corre el grave riesgo de quedar fuera de los procesos sociales académicos, incluso de desertar de las universidades y de sus respectivas carreras 0 de tener graves problemas en sus procesos de titulación.

En el marco de las conclusiones, es necesario indagar sobre el grupo específico del cuerpo docente, para determinar si dentro de su práctica profesional tienen formación en didáctica de la redacción para dirigir a sus estudiantes en el desarrollo de los diferentes géneros discursivos pertinentes a sus cátedras. Además, parece necesario profundizar en el estudio de la alfabetización académica de la muestra con más variables que incluyan los diferentes géneros en los que trabajan los estudiantes.

\section{REFERENCIAS BIBLIOGRÁFICAS}

Adoumieh, N. (2018). El modelo discursivo en la escritura a través del currículo. Revista Caribeña de Investigación Educativa, 77-93. Recuperado el Abril de 2018, de https://www.researchgate.net/ publication/329858045_El_modelo_discursivo_en_la_escritura_a_traves_del_curriculo

Alexopoulou, A. (2011). Del texto a la lengua: la aplicación de los textos a la enseñanza-aprendizaje del español L2-LE. El enfoque basado en los géneros textuales y la evalulación de la competencia discursiva (págs. 97-110). Salamanca: Kadmos. Recuperado el 15 de 02 de 2018, de https://dialnet.unirioja.es/servlet/libro?codigo $=583797$

Álvarez, A. (2005). Escribir en español. Oviedo: Ediuno.
Andueza, A., \& Aguilera, N. (2018). Enseñar y aprender la coherencia textual: una propuesta didáctica a partir de la teoría de Michel Charolles. Didáctica. Lengua y Literatura, 23-40. Recuperado el 20 de 08 de 2019, de https://www.academia.edu/37978641/ Ense\%C3\%B1ar_y_aprender_la_coherencia_textual_una_ propuesta_did\%C3\%A1ctica_a_partir_de_la_teor\%C3\%ADa_de_Michel_Charolles

Bajtín, M. (2011). Las fronteras del discurso. Buenos Aires: Las Cuarenta.

Borsinger De Montemayor, A., \& al., e. (2014). Los textos de la ciencia. Córdova: Comunicarte.

Carlino, P. (2008). Escribir, leer y aprender en la universidad. Buenos Aires: EFE.

Carlino, P. (2013). Alfabetización académica, diez años después. Revista Mexicana de Investigación Educativa, 355-381. Recuperado el Enero de 2018, de https://www.redalyc.org/ pdf/140/14025774003.pdf

Cohen, R. (diciembre de 2007). Las voces de los otros en el discurso académico de los investigadores en la educación. Tesis Las voces de los otros en el discurso académico de los investigadores en la educación. Caracas, Venezuela.

Díaz, Á. (2009). La argumentación escrita. Antioquia: Editorial Universidad de Antioquia.

El Universo. (14 de Noviembre de 2013). Senescyt presume plagio en la mitad de tesis de universitarios. El Universo. Recuperado el 2 de Marzo de 2020, de https://www.eluniverso.com/noticias/2013/11/14/nota/1725496/se-presume-plagio-mitad-tesis

Fregoso, G. (13 de septiembre de 2008). Congreso Iberoamericano de Educación. Universidad Eafit, 9-22. Obtenido de Metas 2021: https://dialnet.unirioja.es/descarga/articulo/4035698.pdf

Gil, J. M. (1999). ProQuestEbookCentral. Obtenido de ProQuestEbookCentral: http://ebookcentral.proquest.com/lib/pucesp/detail. action?doclD=3204914.

Guzman, F., \& García, E. (2015). La alfabetización académica en la universidad. Un estudio predictivo. RELIEVE, 21(1), 1-16. Obtenido de https://ojs.uv.es/index.php/RELIEVE/article/view/5018/6258

Iglesias, T., González, A., \& Hernández, D. (04 de 2019). La progresión temática y la coherencia como criterios textuales en la construcción de párrafos. MENDIVE, 17(2), 293-302. Recuperado el 17 de 10 de 2019, de https://dialnet-unirioja-es.puce.idm.oclc. org/servlet/articulo?codigo $=7050497$

Karam, T. (2005). Una introducción al estudio del discurso y análisis del discurso. Global Medial Journal Edición Iberoamericana, pp. 34-50.

Loureda, Ó. (2010). Nuevas perspectivas para el análisis del texto: una introducción a la lingüística del texto integral. Revista de investigación lingüística, 127-154. Recuperado el Abril de 2018, de https://revistas.um.es/ril/article/view/114161

Mestanza, R. (2018). La reflexión metacognitiva en universitarios como herramienta para mejorar ensayos (tesis de posgrado). México, Morelia, Michoacán: Universidad Michoacana de San Nicolás de Hidalgo. Recuperado el Noviembre de 2020, de http://bibliotecavirtual.dgb.umich.mx:8083/jspui/

Molina, V. (2012). Tensiones entre discursos de estudiantes y profesores universitarios sobre la lectura y la escritura. Signo y Pensamiento, 126-141. Recuperado el 21 de Diciembre de 2018, de https://www.academia.edu/11677340/Tensiones_entre_discursos_de_estudiantes_y_profesores_universitarios_sobre_ la_lectura_y_la_escritura

Núñez, J. (2013). La alfabetización académica: estudio comparado en el ámbito iberoamericano. Madrid. Obtenido de https://repositorio. uam.es/handle/10486/13256 
Perrenoud, P. (2007). Pedagogía diferenciada. Madrid: Popular.

Placci, G., \& Garófolo, A. (2014). Facultad de Ciencias Humanas, Universidad Nacional de Río Cuarto. Facultad de Ciencias Humanas, Universidad Nacional de Río Cuarto (págs. 1-10). Río Cuarto: Universidad de Río Cuarto. Recuperado el Agosto de 2019, de https://core.ac.uk/download/pdf/61704557.pdf

Polo, N. (2012). La voz en el discurso académico. Forma y Función, 25(2), 323-333. Recuperado el 17 de 05 de 2018, de http://www.scielo.org.co/scielo.php?script=sci_abstract\&pi$\mathrm{d}=$ S0120-338X2012000200014

Rojas, I. (2016). Aportes de la lingüística sistémico-funcional para la enseñanza de la lectura y la escritura en la educación superior. Educación y Educadores, 9, 185-204. Recuperado el Febrero de 2019, de https://www.redalyc.org/articulo. oa? id $=83446681001$

Sorókina, T. (2008). El párrafo: las estructuras discursivas. Hesperia. Anuario de Filología hispánica, 1(11), 73-85. Recuperado el 15 de 09 de 2018, de https://dialnet.unirioja.es/servlet/articulo?codigo $=2722466$

UNESCO. (2008). El desafío de la alfabetización en el mundo: UNESCO. Obtenido de UNESCO: https://unesdoc.unesco.org/ark:/48223/ pf0000163170_spa

Villavicencio, M., Carlino, P., Colombo, L., Cordero, G., Molina, E., \& Novillo, M. (2018). La escritura académica y sus vínculos con la docencia, la investigación y el posgrado: experiencias y reflexiones desde la Universidad de Cuenca. Cuenca, Ecuador. Recuperado el 17 de Septiembre de 2018, de https://www.academia. edu/37382610/La_escritura_acad\%C3\%A9mica_y_sus_v\%C3\%ADnculos_con_la_docencia_la_investigaci\%C3\%B3n_y_ el_posgrado_experiencias_y_reflexiones_desde_la_Universidad_de_Cuenca 